\title{
Mental training as a tool in the neuroscientific study of brain and cognitive plasticity
}

\author{
Heleen A. Slagter ${ }^{*}$, Richard J. Davidson ${ }^{2,3,4}$ and Antoine Lutz ${ }^{2}$ \\ Brain and Cognition Unit, Department of Psychology, University of Amsterdam, Amsterdam, Netherlands \\ 2 Waisman Laboratory for Brain Imaging and Behavior, University of Wisconsin, Madison, WI, USA \\ ${ }^{3}$ Department of Psychology, University of Wisconsin, Madison, WI, USA \\ ${ }^{4}$ Center for Investigating Healthy Minds, University of Wisconsin, Madison, WI, USA
}

\section{Edited by:}

Michael X. Cohen, University of

Amsterdam, Netherlands

\section{Reviewed by:}

Michael Posner, University of Oregon, USA

Tonya L. Jacobs, University of

California, Davis, USA

\section{${ }^{*}$ Correspondence:}

Heleen A. Slagter, Department of Psychology, University of Amsterdam, Roeterstraat 15, 1018WB Amsterdam, Netherlands.

e-mail:h.a.slagter@uva.nl
Although the adult brain was once seen as a rather static organ, it is now clear that the organization of brain circuitry is constantly changing as a function of experience or learning. Yet, research also shows that learning is often specific to the trained stimuli and task, and does not improve performance on novel tasks, even very similar ones. This perspective examines the idea that systematic mental training, as cultivated by meditation, can induce learning that is not stimulus or task specific, but process specific. Many meditation practices are explicitly designed to enhance specific, well-defined core cognitive processes. We will argue that this focus on enhancing core cognitive processes, as well as several general characteristics of meditation regimens, may specifically foster process-specific learning. To this end, we first define meditation and discuss key findings from recent neuroimaging studies of meditation. We then identify several characteristics of specific meditation training regimes that may determine process-specific learning. These characteristics include ongoing variability in stimulus input, the meta-cognitive nature of the processes trained, task difficulty, the focus on maintaining an optimal level of arousal, and the duration of training. Lastly, we discuss the methodological challenges that researchers face when attempting to control or characterize the multiple factors that may underlie meditation training effects.

Keywords: plasticity, training, mental training, meditation, neuroimaging, cognition, brain

\section{INTRODUCTION}

The ability to learn is a function of brain plasticity and essential to the survival of all animals. Humans appear remarkable in this respect, as they can acquire a wide range of skills given appropriate training. Neuroscience research within the last few decades confirms that the human brain is plastic, and much more so than once thought possible (Buonomano and Merzenich, 1998). One of the most salient findings is perhaps the observation that the adult brain can still change significantly in both structure and function as a result of experience (e.g., Maguire et al., 2000; Draganski et al., 2004; Boyke et al., 2008). For example, taxi drivers, who obtain extensive navigational experience as adults, have larger posterior hippocampi, a brain structure important for spatial representation of the environment (Maguire et al., 2000). Not only long-term expertise, but also relatively short practice has been associated with neural changes in adults. For example, performing a five-finger piano exercise for $2 \mathrm{~h}$ on five consecutive days resulted in an enlargement of primary motor areas representing the finger muscles, which was accompanied by improved performance (Pascual-Leone et al., 1995, 2005). Remarkably, this study also showed that mental practice of the same finger exercise resulted in a similar reorganization of the motor cortex to the one observed in the group of participants that physically practiced the movements. Thus, plasticity appears to be an intrinsic property of the nervous system retained throughout a lifespan and the obligatory consequence of all neural activity, including mental practice. Indeed, it is now commonly held that the brain undergoes continuous changes in response to each sensory input, motor act, association, reward signal, action plan, and awareness itself (Pascual-Leone et al., 2005).
Yet, research also shows that learning is typically highly specific (Salomon and Perkins, 1989; Kramer and Willis, 2003; Green and Bavelier, 2008; Roelfsema et al., 2010). While individuals trained on a task will improve on that very task, other tasks, even very similar ones, often show little or no improvement. For example, in visual learning tasks, improvements are often not observed if the untrained test stimulus has a different orientation or contrast than the trained stimulus (Roelfsema et al., 2010). Similarly, memory training often enhances memory for the trained content, but not memory skills themselves. In the example of the taxi drivers, follow-up research showed that the taxi drivers did not exhibit improved performance on other memory tasks (Woollett and Maguire, 2009). Thus, training benefits are often stimulus or content specific rather than process specific, a fact that is well documented in the perceptual (Roelfsema et al., 2010), cognitive (Kramer and Willis, 2003), and motor (Bachman, 1961) domain. This is obviously a potentially severe impediment for training programs in educational or clinical settings, where the goal is to improve performance in everyday life and which thus necessarily require a general improvement in skills.

The aim of this paper is twofold: (i) to examine the hypothesis that systematic mental training can induce process-specific learning, that is, learning that generalizes to novel stimuli and task contexts, and (ii) to identify aspects of training contexts that may contribute to process-specific learning from the vantage point of mental training. If plasticity is indeed the obligatory consequence of not only each sensory input or motor act, but also of each thought process, changes in brain circuitry due to mental training 
should lead to process-specific learning. Here, we use the term "process-specific learning" to denote learning effects that do not only improve performance on the trained task or tasks, but also transfer to new tasks and domains (cf. Green and Bavelier, 2008), i.e., learning that is not specific to the trained stimuli or tasks. The main focus of the paper will be on effects of mental training of cognitive control processes. "Cognitive control" refers to a collection of processes that allow us flexibly adapt our behavior in the pursuit of an internal goal, and includes processes such as selection of goal-relevant information, performance monitoring, interference resolution, and storage and manipulation of information in working memory. These processes are generally considered to be largely independent from one another, because they are differentially affected by independent variables, and can be behaviorally and neurally dissociated. There is an emerging consensus in the literature that this conglomerate of largely independent, but constantly interacting control processes is domain general (e.g., Baddeley, 1996; Fuster, 1997; Smith and Jonides, 1999; Miyake et al., 2000). Specifically, the general proposal is that the engagement of a particular cognitive control process (e.g., performance monitoring) within any one cognitive task is simply a matter of the degree to which load is exercised on that control mechanism and should extend to any cognitive challenge that incorporates sufficient control requirements of the same kind. The focus of this paper on the amenability of cognitive control processes to training is thus especially valuable because these abilities are fundamental to higher cognition and contribute to performance across cognitive domains (e.g., attention, working memory, long-term memory; Smith and Jonides, 1999; Duncan and Owen, 2000).

This paper will focus in particular on mental training of cognitive control processes as cultivated by meditation. Little is currently known about the amenability of cognitive control processes to mental training. Most studies to date have focused on mental training in the motor domain (for recent reviews, see, e.g., Guillot and Collet, 2005; Munzert et al., 2009). This is likely related to the fact that it is difficult to design controlled mental training contexts in which an individual can consistently practice a specific cognitive control skill. Yet, being able to enhance cognitive control function through training is especially valuable since, as mentioned above, cognitive control skills, such as the ability to focus attention in face of distraction, contribute to performance on virtually any task (Smith and Jonides, 1999; Duncan and Owen, 2000). Notably, many meditation practices are based on precisely descriptive and highly detailed theories (Gyatso and Jinpa, 1995; Gunaratana, 2007) and are explicitly designed to train such core cognitive skills, and typically in the absence of performing some external task (Lutz et al., 2007, 2008). Furthermore, many of these practices include a focus on maintaining optimal levels of arousal and motivation, and ascending stages through which the practitioner passes, ensuring continuously challenging training conditions. These are all factors that may foster more general learning (e.g., Green and Bavelier, 2008), as will be discussed in more detail below. The neuroscientific study of meditation may thus provide important insights into the potential of mental training to strengthen cognitive control skills, and the factors that contribute to, and the mechanisms that underlie process-specific learning.
The paper is organized as follows. First, to demonstrate the ability of purely mental training to influence brain function and structure, and thereby, behavior, we will briefly review findings from studies of mental practice in the motor domain. We will then focus on mental training in the cognitive domain. To this end, we will introduce standard cognitive-based meditation practices, and discuss recent key findings to illustrate the usefulness of mental training as cultivated by these meditation practices in the neuroscientific study of brain and cognitive plasticity. Next, we will review characteristics of mental training contexts that may promote process-related learning from the vantage point of meditation. In this context, we will also discuss recent findings from studies using other complex training protocols in non-laboratory settings, such as action video gaming, that have reported learning that appears more general than once thought possible. Then, we will discuss methodological challenges that the neuroscientific study of meditation faces in particular. The paper will end with a discussion in which important avenues for future meditation research are identified.

\section{MENTAL TRAINING IN THE MOTOR DOMAIN}

Research has convincingly shown that mental training using motor imagery, like physical practice, can produce changes in brain structure and function that are associated with improved subsequent performance of a motor skill (Guillot and Collet, 2005; Munzert et al., 2009). In a seminal study, mentioned above, Pascual-Leone et al. (1995) showed that mental practice alone is sufficient to promote the modulation of neural circuits involved in the early stages of motor skill learning. Two hours of mental practice of a finger tapping sequence on five separate days was associated with both an enlargement of the representations of the fingers in primary motor cortex, as well as improved motor performance. In another study, Ranganathan et al. (2004) found that strength gains following mental training were related to increases in the amplitude of scalp-recorded brain potentials over midline and lateral motor and midline frontal regions. They proposed that mental training may have affected higher-order motor cortical regions, such as supplementary motor and prefrontal regions, which may in turn have influenced primary motor areas. A recent study using the high spatial resolution of functional magnetic resonance imaging (fMRI) confirmed that gains in motor behavior after mental training are associated with changes in higher-order motor cortical regions (Nyberg et al., 2006). Mental training-related changes in neural activity were observed in the supplementary motor area, as well as in visual cortex. Thus, mental practice of a motor act has been shown to produce changes in brain function and improve performance. Not surprisingly, therefore, mental practice has long found wide acceptance in the training of athletes (e.g., Suinn, 1984).

An important question is how mental training using motor imagery may improve motor performance (Jeannerod, 1996). Behavioral studies have shown that there is a strong correlation between imagined and executed actions along various behavioral dimensions, suggesting that mental and physical training rely in part on common mechanisms (Jeannerod, 1994; Grush, 2004). For example, the time it takes to imagine an action is closely correlated with the execution time of the action (Decety et al., 1989). In line with the idea that the contents of the representations that are formed and strengthened through the two types of practice 
overlap to some extent, neuroimaging research has shown that motor imagery and motor execution activate similar, although not identical, neural networks (e.g., Jeannerod, 1995; Decety, 1996; Lotze et al., 1999; Kosslyn et al., 2007; Munzert et al., 2009). Yet, there are important differences between mental and physical practice. Mental training using motor imagery heavily depends on cognitive aspects of action control, such as motor planning, as well as on working memory to transform, maintain, and inspect information about a motor act. In addition, in contrast to physical practice, although a motor plan is generated, it is prevented from operating on the body (Jeannerod, 1994; Grush, 2004). These differences between mental and motor training are substantiated by neuroimaging research showing that mental and motor training can affect distinct neural networks (Lacourse et al., 2005; Nyberg et al., 2006; Olsson et al., 2008).

It has been proposed that mental training may be more likely to develop and strengthen effector-independent representations, rather than effector-dependent representations, whereas for physical practice, the reverse may be true (Olsson et al., 2008). It is notable in this respect that several studies have reported benefits of mental training using motor imagery on tasks that are largely cognitive (Minas, 1978; Driskell et al., 1994). Of further importance, in some studies, mental practice (Wohldmann et al., 2008), as well as combined mental and motor practice (Olsson et al., 2008) has been associated with greater transfer of learning than physical practice, that is, better performance on novel tasks that call upon the trained skill. These data support the idea that mental practice using motor imagery may strengthen more abstract representations that do not involve specific effectors, thereby fostering process-specific learning that more easily generalizes across stimuli and tasks. Initial evidence for this idea comes from an fMRI study by Olsson et al. (2008), who showed facilitated transfer of learning after combined motor and mental training that was related to changes in the connections from both motor and cognitive systems to the cerebellum.

To conclude, mental training of motor skills leads to changes in brain circuitry and behavior, just as physical training produces changes in brain circuitry and behavior. Yet, the mechanisms underlying mental and physical training of motor skills are not identical. Notably, mental training may improve motor performance by strengthening more abstract representations, and thereby promote transfer of learning to novel task contexts.

\section{MENTAL TRAINING IN THE COGNITIVE DOMAIN}

We will now turn to mental training that is specifically focused at enhancing cognitive function rather than at improving motor performance. Just like processes related to motor control, processes related to the control of cognitive processes should be amendable to mental training. As mentioned in the introduction, we focus here on cognitive control processes, as their amenability to training is especially valuable because these abilities are fundamental to higher cognition and contribute to performance across a wide range of cognitively demanding tasks (Smith and Jonides, 1999; Duncan and Owen, 2000). Yet, unfortunately, relatively little is known about the amenability of such processes to mental training. As mentioned above, this is likely due to the fact that it is difficult to design controlled mental training contexts. Notably, many meditation practices are based on precisely descriptive and highly detailed theories and are explicitly designed to train specific, well-defined cognitive control skills. The neuroscientific study of meditation may thus provide important insights into the usefulness of mental training as a tool in the study of brain and cognitive plasticity (see also Tang and Posner, 2009; Xiong and Doraiswamy, 2009).

Despite a large number of scientific reports and theoretical proposals, the neurophysiological processes involved in meditation and the long-term impact of meditation on the brain and cognitive function are still largely unknown. The lack of statistical evidence, control populations and tasks, and the heterogeneity of the studied meditative states can, in part, account for the limited contributions made by neuroscience-oriented research on meditation. Therefore, rather than providing an exhaustive review of this literature, we will only discuss recent key findings that speak to the idea that mental training of cognitive skills can induce process-specific learning. Other articles have recently reviewed this literature more exhaustively (see, e.g., Cahn and Polich, 2006; Lutz et al., 2007). Specifically, in the below, we will first define meditation, and review recent key findings from neuroimaging studies of meditation. Modern neuroimaging techniques provide a new possibility for understanding process-specific learning by revealing its underlying mechanisms. We will then identify aspects of mental training as cultivated by meditation that may foster process-specific learning.

\section{MEDITATION}

The term "meditation" refers to a broad variety of practices, ranging from techniques designed to promote relaxation or improve attentional function to exercises performed with more far-reaching goals such as a heightened sense of well-being or the cultivation of altruistic behaviors. Not all practices thus focus on explicit training of specific cognitive skills, and it is therefore essential to be specific about the type of meditation practice under investigation. Here we focus on two common styles of Buddhist contemplative techniques, because (1) Buddhist traditions offer extensive, precisely descriptive and highly detailed theories about these practices in a manner that lends itself readily to appropriation into a neuroscientific context (Gyatso and Jinpa, 1995; Gunaratana, 2007; Lutz et al., 2007), and (2) these techniques are now being applied in clinical settings (e.g., Kabat-Zinn et al., 2000; Teasdale et al., 2000). These two meditation styles are often combined, whether in a single session or over the course of practitioner's training, and are explicitly designed to train specific cognitive processes. The first style, focused attention (FA) meditation, entails voluntary focusing attention on a chosen object in a sustained fashion, such as a visual object, a visualized image, or breath sensations (Lutz et al., 2007, 2008). To sustain this focus, the practitioner must also constantly monitor and regulate the quality of attention. Thus, FA meditation is thought to not only train one's ability to sustain attention, but also to develop three regulatory skills; the first is the monitoring faculty that remains vigilant to distractions without destabilizing the intended focus. The next skill is the ability to disengage from a distracting object without further involvement. The last involves the ability to redirect focus promptly to the chosen object. One may note remarkable parallels between the processes involved in FA meditation, as described above, and recent cognitive (neuro)science conceptualizations of attention. Western scientists also recognize that the ability to focus and sustain attention on an intended object requires skills involved 
in monitoring the focus of attention and detecting distraction, disengaging attention from the source of distraction, and (re)directing and engaging attention to the intended object (e.g., Posner and Petersen, 1990).

The second style of meditation, open monitoring (OM) meditation, involves non-reactively monitoring the content of experience from moment-to-moment, without focusing on any explicit object (Lutz et al., 2007, 2008). OM meditation typically starts by calming the mind and reducing distractions using FA meditation. The practitioner then gradually reduces the focus on an explicit object in FA, and the monitoring faculty is correspondingly emphasized. Usually, there is also an increasing emphasis on cultivating a "reflexive" awareness that grants one greater access to the rich features of each experience, such as the degree of phenomenal intensity, the emotional tone, and the active cognitive schema. OM meditation is thought to enhance non-reactive meta-cognitive monitoring, as well as increase awareness of automatic cognitive and emotional interpretations of sensory, perceptual, and endogenous stimuli, and thereby cognitive flexibility and reappraisal (Bishop et al., 2004; Lutz et al., 2008; Chambers et al., 2009).

Both FA and OM meditation are assumed to induce a predictable and distinctive state (or set of states) whose occurrence is clearly indicated by certain cognitive and emotional features. These states, which arise during practice and are relatively short-term, can allegedly result in enduring changes in mental function, i.e., in the development of certain traits (Lutz et al., 2007, 2008). For example, FA meditation is said to develop, as one advances, the three regulative skills to the point that, for example, advanced practitioners have an especially acute ability to notice when the mind has wandered. Eventually, this induces a trait change, whereby the attention rests more readily and stably on the chosen focus. At the most advanced levels, the regulative skills are invoked less and less frequently, and the ability to sustain focus thus becomes progressively "effortless." It should be noted that this explicit focus on inducing a particular state of mind (or set of mental states) comprises an important difference between meditation practices, like FA and OM, and computerized task training of cognitive function. While computerized task training might also lead to changes in mental state (e.g., being focused), this is typically not its primary aim. To summarize, FA and OM practices are designed to train specific mental processes and such changes are thought to gradually endure through time, i.e., result in process-specific learning and the development of traits.

\section{NEUROIMAGING STUDIES OF MEDITATION}

Although the systematic study of meditation is still in its infancy, recent findings suggest that experience in FA and OM meditation is associated with changes in brain and cognitive function both during meditation and during performance of external tasks that do not require meditation (Cahn and Polich, 2006; Lutz et al., 2007, 2008; Chambers et al., 2009). Neuroimaging studies examining the neural correlates of meditation indicate that task demands of the two meditation styles are reflected in characteristic neural activity patterns. For example, a recent cross-sectional study found that FA meditation on an external visual point compared to a rest condition was associated with greater activation in multiple brain regions, implicated in attentional processing, including the superior frontal sulcus and the intraparietal sulcus, in long-term meditation experts compared to novices (Brefczynski-Lewis et al., 2007). More importantly, neuroimaging work suggests that FA and OM meditation may not only during meditation activate brain regions involved in the psychological processes that are allegedly evoked, but may also produce lasting changes in brain and mental function that translate to improved performance in novel task contexts. For instance, several recent studies of FA meditation have reported improvements in sustained voluntary attention during performance of external tasks that do not require meditation. In one longitudinal study, in which participants were randomly assigned to a meditation-retreat group or a waiting-list control group, $5 \mathrm{~h}$ /day of meditation practice that involved voluntary focusing attention on a chosen object in a sustained fashion for 3 months was found to improve sustained visual attention (MacLean et al., 2010). Specifically, compared to the waiting-list control group, meditation-retreat participants showed enhanced perceptual discrimination and vigilance on a sustained visual attention task requiring participants to discriminate a rare threshold-level short line from a standard long line. Notably, these enhancements in sustained attention ability were still observed 3 months after the retreat ended, demonstrating enduring changes in sustained attention. In another recent longitudinal study, Lutz et al. (2009) found that 3 months of intensive FA and OM meditation was associated with enhanced moment-to-moment stability of attention during FA meditation, as measured both by reduced response time variability to rare target events in an auditory sustained attention task (Figure 1A) and increased trial-totrial consistency of oscillatory neural responses over frontal scalp regions to these events as recorded with electroencephalography (EEG; Figure 1B). Furthermore, those individuals who showed the greatest training-induced increase in neural response consistency showed the largest decrease in behavioral response variability (Figure 1C). In addition, this study reported that intense mental training affected cortical engagement, as reflected by a concomitant reduction in event-related desynchronization (ERD) to target tones (Figure 1D). ERD is usually viewed as a correlate of increased cellular excitability in thalamocortical systems during cortical information processing (Pfurtscheller and da Silva, 1999). Within this framework, previous studies have interpreted reductions in ERD after practice of external tasks as decreased cognitive effort (Pfurtscheller and da Silva, 1999; Romero et al., 2008). A mental training-related reduction in task effort would be consistent with traditional accounts of progress in this practice (Gyatso and Jinpa, 1995; Gunaratana, 2007). In these accounts, the regulative attentional skills allegedly are invoked less and less frequently with more advanced level of training, and the ability to sustain focus is said to become progressively "effortless." Since these effects were observed over time within the same individual and during external task performance, together the above findings suggest that purely mental training of FA generalizes to improvements in performance on novel tasks that call upon the trained skills. These longitudinal findings also indicate that the enhanced sustained attention ability most likely reflects plasticity in the adult brain, and more generally, that attentional skills are subject to training.

Long-term practice of OM meditation is also thought to result in enduring changes in cognitive and brain function. Specifically, because OM meditation fosters non-reactive awareness of the stream of experience without deliberate selection of a primary 


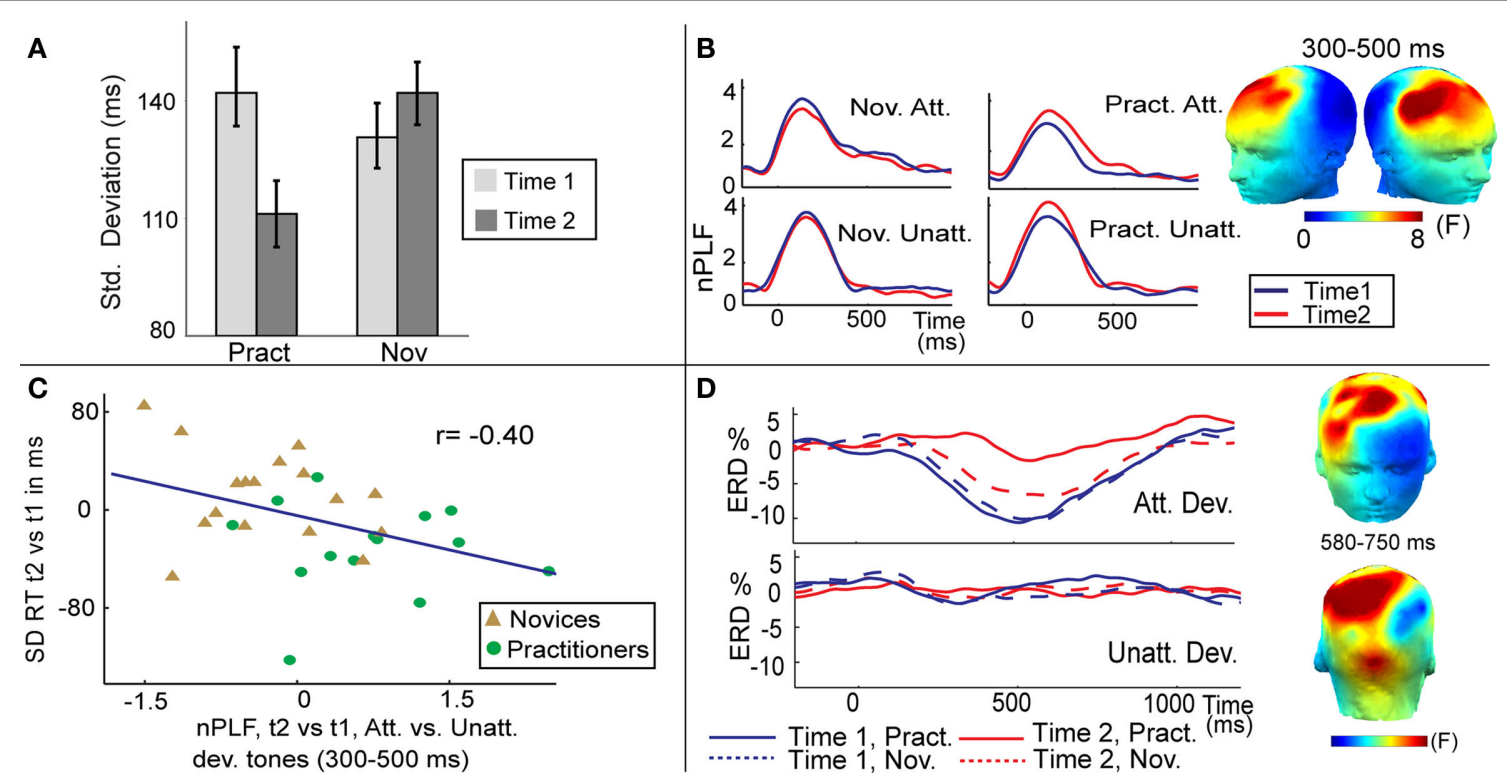

FIGURE 1 | Effects of FA meditation. (A) Intensive mental training reduced intra-individual variability of behavioral performance. Average SD of reaction time in response to target (attended deviant) tones, separately for each session (time 1, time 2) and group [practitioners (Pract), novices (Nov)]. (B) Intensive mental training increased trial-to-trial consistency of brain responses to attended deviant tones. The scalp maps show the spatial distribution of the mental training-related increase in theta-band $(4-7 \mathrm{~Hz})$ phase consistency (indexed by normalized PLF) to target tones, as indexed by a three-way interaction between group, session, and condition (attended, unattended deviant tones). Fvalues are averaged between 300 and $500 \mathrm{~ms}$. The time course of normalized PLF values averaged across the electrodes sites showing this significant three-way interaction is shown separately for each group, session, and attended (Att) and unattended (Unatt) deviant tones. Note that the observed increase in phase consistency to attended deviant tones over time was only observed for the practitioner group. (C) The correlation plot shows that the observed change in the trial-to-trial variability of brain responses predicted the observed behavioral change in the trial-to-trial variability of the RT. (D) Mental training selectively reduced cognitive effort as indexed by ERD. Time course of the ERD for attended and unattended deviant tones (Dev.) shown for the practitioners and novices separately. The scalp maps show the spatial distribution of the mental training-related decrease in beta-band $(13-30 \mathrm{~Hz})$ ERD to attended vs. unattended deviant tones [three-way interaction between group, session, and condition]. Fvalues are averaged between 580 and $750 \mathrm{~ms}$. Figure is adopted from Lutz et al. (2009). object, intensive practice can be expected to reduce the elaborative thinking that would be stimulated by evaluating or interpreting a selected object (Cohen et al., 1996; Lutz et al., 2007, 2008). In line with this idea, Slagter et al. $(2007,2009)$ recently found that 3 months of intensive OM meditation reduced elaborative processing of the first of two target stimuli (T1 and T2) presented in a rapid stream of distracters, as indicated by a smaller T1-elicited P3b, a brain potential index of resource allocation (Figure 2A). Moreover, this reduction in resource allocation to $\mathrm{T} 1$ left neural resources more rapidly available for $\mathrm{T} 2$, as indexed by an increase in trial-to-trial phase consistency of neural oscillatory activity in the theta frequency range (Figure 2B), and was associated with improved detection of T2 when it followed T1 within half a second (Figure 2C). Because participants were not engaged in formal meditation during task performance in this study, these results provide support for the idea that one effect of an intensive training in OM meditation might be a general reduction in the propensity to "get stuck" on a target, as reflected in less elaborate stimulus processing, and the development of efficient mechanisms to engage and then disengage from target stimuli in response to task demands. OM meditation is also thought to cultivate a reflexive awareness of the subjective features of a given moment, such as its emotional tone, by engaging processes involved in interoception or awareness of bodily responses (Lutz et al., 2008). In line with this idea, a recent fMRI study found greater activity in network of brain areas involved in interoception, including the anterior insula and the somatosensory cortex, during a monitoring state relative to a narrative generation state in participants who were randomly assigned to an 8-week course incorporating OM meditation compared with a group of waitlist controls (Farb et al., 2007).

Next to these functional changes, several studies have also reported structural changes in the brain of long-term FA and OM meditators compared to novices in regions that are typically activated during these meditations (Lazar et al., 2005; Hölzel et al., 2008; Grant et al., 2010). For example, a study by Hölzel et al. (2008) found greater gray matter concentration in the anterior insula, a brain region, as mentioned above, important for awareness of internal experience, in experienced Vipassana meditators (mean practice: 8.6 years; $2 \mathrm{~h}$ daily). In another study, more than $1000 \mathrm{~h}$ of Zen meditation was associated with increased cortical thickness in the dorsal anterior cingulate, a region important in the adaptive control of behavior, and bilaterally, in the secondary somatosensory cortex. It is important that these cross-sectional findings are supplemented by findings from prospective studies to show that these structural changes result from meditation rather than, e.g., preexisting individual differences. In addition, because both Vipassana and Zen meditation include forms of FA and OM meditation, future studies are necessary to determine how FA and OM meditation may each contribute to these anatomical changes. 


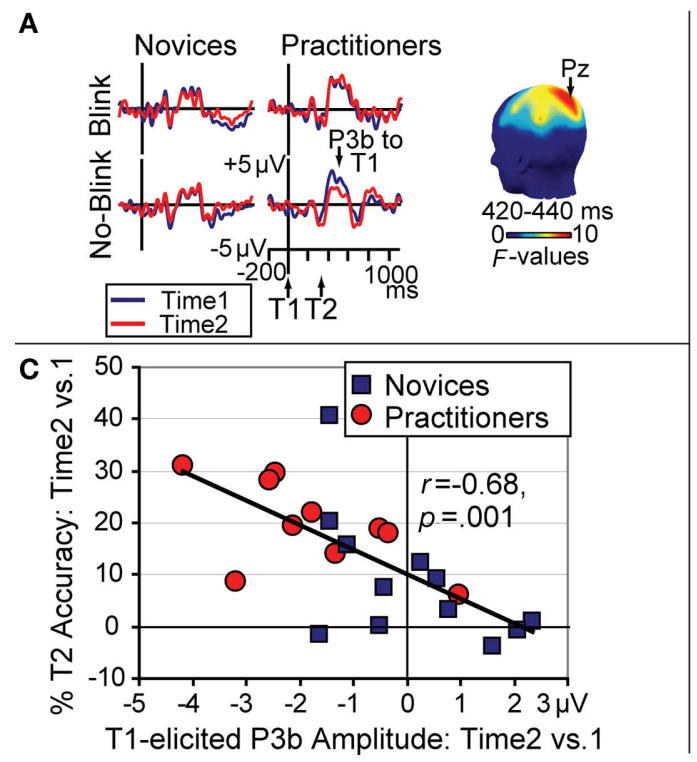

FIGURE 2 | Effects of OM meditation. (A) Brain potentials from electrode Pz, time-locked to T1 onset in short-interval trials as a function of session (time 2 vs. 1), T2 accuracy (no-blink vs. blink), and group (practitioners vs. novices). This figure panel illustrates that intensive OM meditation was associated with a selective reduction in T1-elicited P3b amplitude, a brain potential index of resource allocation, in no-blink trials in the practitioner group. The scalp map shows electrode sites where this three-way interaction between session, T2 accuracy, and group was significant between 420 and $440 \mathrm{~ms}$. Adopted from Slagter et al. (2007); (B) Target locking of the theta frequency band phase at electrodes Fz and FT8, time-locked to T1 onset, shown for short-interval no-blink trials and separately for each session and group. Neural activity in the theta frequency band phase-locked robustly to consciously perceived target stimuli over frontal scalp regions. Importantly, a significant meditation-related increase in T2 phase
B

Theta (4.1-8.5Hz) Phase Consistency in No-Blink trials
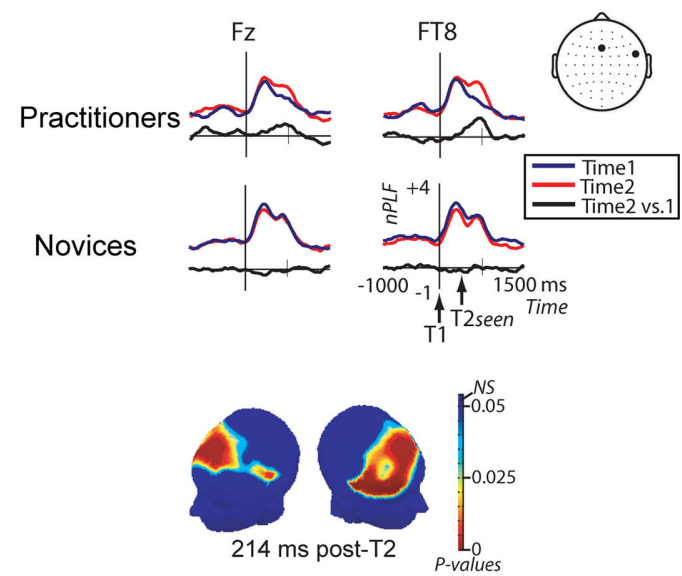

Group by Time Interaction
To summarize, mental training of cognitive skills as cultivated by FA and OM meditation has been associated with changes in brain structure and function, as well as improved task performance. These findings provide initial support for the idea that systematic mental training of cognitive skills, as cultivated by meditation, can improve performance on external tasks that call upon the trained skills, and hence can strengthen specific cognitive processes. These findings also add to a growing body of literature demonstrating plasticity in the adult brain, and may provide initial insights into the basic mechanisms that underlie cognitive process-specific learning.

\section{FACTORS THAT DETERMINE PROCESS-SPECIFIC LEARNING}

A long-standing issue in cognitive training is how to get training benefits to generalize to different stimuli and tasks (e.g., Schmidt and Bjork, 1992; Kramer and Willis, 2003; Green and Bavelier, 2008). As mentioned above, transfer of learning to novel task contexts has generally been the exception rather than the rule. Yet, purely mental training, as cultivated by meditation, as discussed above, appears to improve performance on novel, external tasks that call upon the trained skills but do not require meditation, and hence, induce process-specific learning. An important question is therefore what aspects of the meditation context may promote process-specific learning. In the below, we will discuss several factors that appear particularly important determinants of process-specific learning from the vantage point of mental training as cultivated by meditation. It should be noted that these factors are not specific to meditation training contexts, and likely represent common determinates of more generalized learning (e.g., Schmidt and Bjork, 1992; Ahissar and Hochstein, 2004; Green and Bavelier, 2008; Ahissar et al., 2009). Other non-laboratory training settings that appear to produce more general learning, such as action video gaming and musical training, also embody many of these characteristics (see Green and Bavelier, 2008 for an extensive review).

\section{COMPLEXITY OF THE TRAINING CONTEXT}

From the above descriptions of FA and OM meditation, it is clear that each of these practices taps several cognitive skills in parallel. For example, FA meditation involves processes involved in directing and sustaining attention on a selected object (e.g., breath sensation), detecting mind wandering and distractors (e.g., thoughts), and disengagement of attention from distractors and shifting of attention back to the selected object. This is a key difference between mental training as cultivated by meditation and typical laboratory training settings that generally have been designed to train one specific cognitive skill, but not others. Like-wise, action video gaming, which has been shown to promote process-specific learning (e.g., Green and Bavelier, 2003, 2006; Donohue et al., 2010), naturally taps into many processes in parallel (Green and Bavelier, 2008). 
Many action video games require players to simultaneously focus attention on task-relevant information, while ignoring distracting events, to monitor the environment and performance, and to track multiple moving objects. Thus, the complexity of training setting may be an important determinant of process-specific learning.

\section{STIMULUS AND TASK VARIABILITY}

Another important determinant of process-specific learning appears to be variability of input and task (Schmidt and Bjork, 1992). High variability at the level of the exemplars or movements to be learned, and the context in which they occur likely prevents learning at the level of specific stimuli/effectors, or stimulus-response relationships, thereby fostering learning at a higher or more abstract level of representation (Seidler, 2004; Green and Bavelier, 2008). It is notable in this respect that the observed structural brain changes in taxi drivers have not been observed in bus drivers, who operate along a constraint set of routes, unlike taxi drivers, who navigate widely around the city (Maguire et al., 2006). Of further importance, although many studies have reported lack of transfer of training benefits to new task contexts, recent studies of external task training in laboratory settings have reported improvements in cognitive task performance and transfer of learning to novel cognitive tasks when the training used a battery of tasks that all called upon the same (to-be-trained) cognitive skill, but involved different stimuli and task contexts (Olesen et al., 2004; Dahlin et al., 2008; Jaeggi et al., 2008; Persson and Reuter-Lorenz, 2008). For example, Dahlin et al. (2008) used six different training tasks, which all required working memory updating, a basic cognitive control skill. Five weeks of training resulted in enhanced performance not only on the trained task, but importantly also on a novel task, which required updating, but differed in terms of memorial content, set size, presentation rate, and response format. This transfer effect was mediated by the striatum, a subcortical brain structure important for updating. Thus, variability of input and task appears to be an important determinant of process-related learning and therefore, whether or not transfer of learning will occur. Mental training as cultivated by FA and OM meditation naturally includes many stimuli of various type and domain (e.g., auditory/somatosensory, cognitive/emotional, internal/external) that occur in different mental contexts. For example, OM meditation consists in being attentive moment by moment to anything that occurs in experience, whether it be a sensation, thought, or feeling. Such variability in stimulus and mental content may contribute to process-specific learning as a function of meditation.

\section{TYPES OF PROCESSES TRAINED}

It has been proposed that cognitive learning is most likely to occur when training programs focus on strengthening cognitive control functions that orchestrate thoughts and actions to make them consistent with internal goals (Persson and Reuter-Lorenz, 2008). As mentioned above, these include processes such as focusing attention, meta-cognitive monitoring, and switching between tasks. These processes are shared by many tasks and are widely viewed as amodal (Smith and Jonides, 1999; Duncan and Owen, 2000). Benefits of training programs focusing on such core cognitive skills may therefore more easily extend across materials and stimulus modalities, and hence, across tasks. Notably, FA and OM meditation are designed explicitly to train core cognitive skills (see above). This may provide another factor that fosters process-specific learning in meditation.

\section{TASK DIFFICULTY}

Learning is more likely to occur when the task remains challenging, albeit not too difficult, throughout the training (Ahissar and Hochstein, 2004). This is the case for example, in action video gaming, where the player advances from one level of difficulty to the next as expertise develops (Green and Bavelier, 2008). This is also true for meditation, where a practitioner is gradually introduced to more refined aspects of a technique while he or she gains more and more familiarity with the practice. Indeed, many contemplative traditions speak of ascending stages through which the practitioner passes, and a single practice may progress gradually through a number of meditative states, that might significantly differ from each other both phenomenally and in terms of the appropriate technique to be applied (Lutz et al., 2007). This progression ensures that the practice remains engaging and may also contribute to process-specific learning.

\section{AROUSAL AND MOTIVATION}

Arousal and motivation are important principles guiding learning. It is well known that training paradigms that lead to low levels of arousal will tend to lead to low amounts of learning, as will training paradigms that lead to excessively high levels of arousal (Frankenhaeuser and Gardell, 1976). Between these extremes is some level of arousal that leads to a maximum amount of learning (Green and Bavelier, 2008). Notably, central to both FA and OM meditation is maintenance of an optimal level of arousal or alertness via the regulation of attention or emotions (Lutz et al., 2007). For example, in most styles of FA meditation, after having placed the attention on the object, the practitioner seeks to avoid two overall "flaws" that represent under- and over-arousal. The first, dullness, is detected, in its most gross form, as a sensation of drowsiness. The second, excitement, manifests itself in distraction or attention wandering to other mental events. When suboptimal levels of arousal are detected, the practitioner can apply counteracting methods. For instance, to counteract drowsiness, the practitioner may add intensity to a visualized object or tension to the body. There are a variety of findings in the literature that already suggests that autonomic changes occur during FA and OM meditations. A frequent finding is a decrease in arousal during these practices (Cahn and Polich, 2006; Lutz et al., 2007). For instance, Takahashi et al. (2005) in a study of Zen meditation found changes in heart rate variability (reflecting parasympathetic nervous system activity) that were associated with changes in specific EEG oscillatory activity patterns. Yet, little is still known on the effect of FA and OM meditations on the maintenance of an optimal balance between excitement and dullness.

Like actively monitoring and regulating arousal levels, motivation is an inherent feature of many meditation practices. A formal meditation session will often begin and end with deliberately invocating some forms of soteriological or altruistic motivations (Gyatso and Jinpa, 1995; Gunaratana, 2007). Such deliberate practice, typically on a daily basis, is known to be a critical factor for the continued improvement of performance (Ericsson, 1996; 
Ericsson and Lehmann, 1996). This feature, as well as the active monitoring and regulation of arousal levels, may be an additional aspect of meditation regimens important for inducing processspecific learning.

\section{DURATION OF TRAINING}

In most laboratory manipulations cognitive training typically is no longer than several weeks, and total training duration in most previous neuroimaging studies of cognitive task training amounted to less than $20 \mathrm{~h}$, even in studies demonstrating transfer of learning (e.g., Dahlin et al., 2008). This duration of training might be too short to induce long-lasting process-specific learning. Indeed, the attainment of an expert level of performance requires about $10,000 \mathrm{~h}$ of deliberate practice in the case of non-laboratory, domain-specific learning, such as performance of chess, surgery, music, and sports (Ericsson and Lehmann, 1996). Because the traditional duration of training in meditation also involves many hours of intensive practice over years (Lutz et al., 2007), the study of longterm meditators may provide a unique opportunity to investigate the development of process-specific learning. Reported differences in brain function between meditation experts ( $>10,000 \mathrm{~h}$ in life) vs. control subjects highlight the ability of meditation experts to generate new data that might not exist without such sustained mental training (Lutz et al., 2004; Carter et al., 2005; Brefczynski-Lewis et al., 2007). These data will need to be supplemented with data from studies that examine meditation-related changes over long time periods within the same individuals, from novice to expert. Such longitudinal data are necessary to exclude the possibility that observed training effects are due to pre-existing differences between groups (i.e., experts and novices) and will allow for a more precise delineation of the developmental trajectory of the trained abilities and the mechanisms that underlie process-specific learning. Thus, duration of training may represent a final determinant of processspecific learning.

Meditation has both short-term, intermediate, and long-term effects. Many studies have reported changes in cognitive task performance after relatively brief practice in the order of several days to weeks (e.g., Jha et al., 2007; Tang et al., 2007, 2010) or after a training period of several months (e.g., MacLean et al., 2010; Sahdra et al., in press). The extent to which these shorter-term changes are enduring and reflect lasting changes in brain function or structure is still unclear. Furthermore, the relationship between short-and long-term effects of meditation is at this point unknown, and longitudinal studies, that follow the same individual over time over longer time periods (i.e., years), are necessary to clarify this relationship.

\section{METHODOLOGICAL CHALLENGES}

The problem of determining whether a treatment or intervention has an effect is ubiquitous in science, and the golden standard is to evaluate effects using randomized, double-blind experimental designs, in which participants are randomly assigned to either the experimental group or a control group, and neither the participants nor the experimenters know which group the participants are in. The neuroscientific study of meditation faces several methodological challenges in particular related to the fact that randomized assignment is not always possible, as in cross-sectional studies comparing meditation experts with non-meditators or less-experienced meditators, and the fact that it is impossible to blind participants to the nature of the study (in this case, meditation). Researchers should therefore be sensitive to potential confounding effects of motivation, demand characteristics, and outcome expectations on study outcomes. For example, participants with an interest or belief in meditation may be more likely to consent to participate and more motivated to follow the study procedure. They may also more strongly believe in positive study outcomes. It is well known that such non-specific factors can significantly influence study outcomes (e.g., Baskin et al., 2003).

One way to deal with these issues is to choose a control group with a clear conception of the research question and the hypothesized mechanism, and thorough consideration of how threats to validity may be best addressed for a given, clearly defined training procedure. For instance, a study may benefit from using an active control group that receives a structurally comparable, but different treatment than the meditation group, for example a physical exercise program (e.g., MacCoon et al., 2011). An active control group controls not only for factors related to the fact that participants cannot be blinded to the meditation intervention, such as expectancy effects, but also for other non-specific factors, such as social interactions, attention given by instructors, and time spend in the study. In studies of meditation to date, including most of the studies reviewed in this article, active control groups have been very rarely used. If we ultimately wish to attribute the changes observed in studies of meditation-based interventions to the active ingredient of meditation per se rather than the many non-specific factors, it is imperative to utilize active control groups that permit such a rigorous comparison. The state of research in this area is still in its infancy, but as the field moves forward, it will be increasingly important to use rigorous comparison groups to which participants are randomly assigned. Of course, in studies of long-term practitioners, this is not possible, but these studies need to be supplemented with longitudinal studies in less-experienced individuals where changes over time can be tracked (see below).

Next to choosing an appropriate control group, inclusion of a proper control task or task condition for which one does not expect to find meditation-related improvements, may also control for some of the above variables, in particular motivation and expectancy effects. For instance, based upon theoretical models of the attentional blink, we predicted that 3 months of Vipassana meditation, a form of OM meditation, (1) would selectively modulate later brain processes related to attentional resource allocation, but not early, sensory-driven brain processes (as indicated by early visual brain potential components), and (2) would only affect T2 processing, not $\mathrm{T} 1$ processing, in trials in which $\mathrm{T} 2$ followed $\mathrm{T} 1$ within the time-window of the attentional blink (in short-interval, but not long-interval trials; Slagter et al., 2007; see above). As predicted, we only found meditation-related improvements in T2 performance in short-interval trials that were associated with selective changes in higher-order T2 processing. Such a theoretically founded, selective effect is difficult to explain in terms of, for example, a placebo effect. Yet, it should be noted that alternate explanations, such as changes in working memory capacity, mood, or arousal, cannot be fully excluded in this study. For example, behavioral studies have reported a relationship between working memory capacity 
and attentional blink size (Colzato et al., 2007), as well as between mood and arousal and the size of the attentional blink (Olivers and Nieuwenhuis, 2006; Jeffries et al., 2008). It is thus possible that OM meditation in this study affected the size of the attentional blink by modulating one of these factors next to, or rather than by training attentional processes itself. This example illustrates the need for future research to determine the precise mechanisms that underlie effects of FA and OM meditation on cognitive phenomena, such as the attentional blink, and their underlying brain circuitry. This research would benefit from including questionnaires and/or tests that measure changes in, e.g., mood and working memory capacity, as well as psychophysiological measures of mood and arousal, such as skin conductance and heart rate variability, to shed further light on the precise mechanisms that may underlie observed effects, and to better control for possible confounding variables.

To summarize, since individuals cannot be blinded with respect to the fact that they are participating in a meditation study, control groups and tasks are essential for validating the effects of meditation. Ideally, participants are randomly assigned to a practitioner and control group, and groups are matched in all aspects other than the factor of interest, most importantly in age, gender, race, socioeconomic status intelligence, motivation and study outcome expectancies. Questionnaires and psychophysiological measures may aid in interpreting observed effects of meditation.

\section{CROSS-SECTIONAL VS. LONGITUDINAL STUDIES}

There are two fundamental strategies for examining the effects of mental training, that each have distinct advantages and disadvantages: cross-sectional and longitudinal approaches. In the crosssectional approach, individuals with varying levels of a given skill are compared and differences in some variables related to their skill level are identified. For example, neural function of long-term practitioners is compared with that of matched control subjects or less-experienced practitioners. This approach is useful for studying mental training-related changes over longer time periods (e.g., many years), where the longitudinal study of individuals may be difficult. Yet, as randomized group assignment is not possible in this approach, cross-sectional designs may suffer from cohort effects, in which different groups (i.e., expert vs. novice meditators) differ from each other by factors of no-interest, for instance in sleep or nutritional habits, or with respect to pre-existing differences in personality characteristics. For example, although lower neuroticism scores are reported by QiGong meditators, who have practiced for a greater number of years (Leung and Singhal, 2004), meditation training is more likely to be discontinued by people with higher trait neuroticism (Delmonte and Kenny, 1985; Delmonte, 1988).

The longitudinal approach studies the same individual at every point. This approach therefore does not necessarily suffer from cohort effects, and provides optimal power to identify trainingrelated changes, because within-subject variability is typically smaller than across-subject variability. Yet, spending a number of months in a retreat environment, as is the case in many longitudinal meditation studies, including some of the studies discussed above (Slagter et al., 2007, 2009; Lutz et al., 2009; MacLean et al., 2010), also brings with it changes in for instance, sleep-wake cycle, mood, and arousal, and may require participants to practice in silence and with their eyes closed, depriving the senses of their usual stimulation. These confounding factors may well influence results. For example, it has been proposed that reduced sensory load does not only affect low-level sensory stimulus processing, for example, by increasing visual cortex excitability (e.g., Boroojerdi et al., 2000; Pitskel et al., 2007) or by modifying loudness perception (e.g., Formby et al., 2003; Munro and Blount, 2009), but can also lead to changes in higher-order cognitive information processing (e.g., Mahon and Caramazza, 2008). Most meditation practices are performed either with defocused open eyes, defocused halfopen (hooded) eyes, or with closed eyes. It is possible that intensive meditation with defocused (half-) open eyes by reducing sensory load causes changes in the visual cortex similar to sensory deprivation. Notably, the longitudinal study by MacLean et al. (2010), discussed above, reported decreased visual perceptual threshold after 3 months of intensive meditation, and only found improved sustained attention task performance when retreat participants were allowed to use their pre-retreat visual stimuli. Improvements in sustained attention were not observed when perceptual thresholds were individually adjusted post-retreat to match each participant's pre-retreat perceptual threshold in difficulty. In this study, meditation-related reductions in sensory load may thus in part explain the observed changes in attentional function. The confound of reduced sensory load during some meditative practices is one that needs to be addressed in future studies. Neuroimaging measures may also be useful in this respect. For example, in the aforementioned Slagter et al. (2007) study, meditation was not associated with changes in early sensory processing (as indexed by the early visual brain potential components $\mathrm{P} 1$ and N1), suggesting that reduced sensory load likely did not contribute to the observed meditation-related changes in higher-order target processing in this study.

To summarize, meditation researchers should be circumspect of possible confounds related to the fact that individuals cannot be blinded to the meditation intervention, confounds that are associated with using cross-sectional and longitudinal study designs, and caveats, such as sensory deprivation or changes in sleep-wake cycle, that are more specific to the study of meditation. The above emphasizes the importance of conducting single-blind meditation studies. Those involved in data collection and analysis should be blinded with regard to group assignment and/or the specific study predictions to prevent experimenter biases (the favoring of certain outcomes over others) from influencing results. Of course, this is not possible in all cases, for example, when participants are studied in retreat settings.

It should be emphasized that many of the above challenges are not unique to the study of meditation. For example, one should always insure that effects of cognitive training cannot be explained by differences in motivation, arousal, mood, or expectancy bias between training and control groups, or by pre-existing differences between groups in age, intelligence, etc. Thus, in general, any cognitive training study should be designed with a clear conception of the research question and the hypothesized mechanism, and thorough consideration of how threats to validity may be best addressed for a given, clearly defined training procedure. Nevertheless, the development of adequate comparison groups against which to compare mental training as cultivated by meditation remains an important avenue for future research. Only when 
done in a scientifically rigorous way can the study of meditation advance our understanding of the plasticity of cognitive processes and their underlying neural circuitry.

\section{DISCUSSION AND CONCLUSION}

In this paper, we have reviewed the rationale for using mental training to study brain and cognitive plasticity. We have illustrated how the application of meditation in conjunction with neuroimaging methods has been used to shed light on the amenability of cognitive functions and their underlying brain circuitry to training. We have also identified several factors that may determine process-specific learning from the vantage point of mental training as cultivated by meditation, and discussed methodological challenges that the study of meditation needs to address. While the neuroscientific study of meditation is clearly still in its infancy, the initial findings reviewed here promise both to reveal the mechanisms by which such training may exert its effects and underscore the plasticity of the brain circuits that underlie complex mental functions. More generally, these findings support the idea that the nervous system is a continuously changing structure of which plasticity is an integral property and the obligatory consequence of not only sensory and motor processing, but also of more complex mental activities, such as focusing attention and meta-cognitive monitoring (Buonomano and Merzenich, 1998; Pascual-Leone et al., 2005). Just as a specific physical exercise will produce selected changes in brain circuitry and performance (Hillman et al., 2008), a specific mental exercise will lead to selected changes in brain circuitry that can significantly affect information processing and behavior.

Building upon previous work (Schmidt and Bjork, 1992; Ahissar and Hochstein, 2004; Green and Bavelier, 2008; Ahissar et al., 2009), we discussed several characteristics of meditation that may be responsible for process-specific learning, including the fact that typically multiple processes are trained in parallel (i.e., context complexity), variability in stimulus input, the manner in which task difficulty is progressed, the focus on maintaining an optimal level of arousal, the motivational state of the learner, and the duration of training. These features likely represent common principles of

\section{REFERENCES}

Ahissar, M., and Hochstein, S. (2004). The reverse hierarchy theory of visual perceptual learning. Trends Cogn. Sci. 8, 457-464.

Ahissar, M., Nahum, M., Nelken, I., and Hochstein, S. (2009). Reverse hierarchies and sensory learning. Philos. Trans. R. Soc. Lond. B Biol. Sci. 364, 285-299.

Bachman, J. C. (1961). Specificity vs. generality in learning and performing two large muscle motor tasks. Res. Q. 32, 3-11.

Baddeley, A. (1996). Exploring the central executive. Q. J. Exp. Psychol. 49, 5-28.

Baskin, T. W., Tierney, S. C., Minami, T., and Wampold, B. E. (2003). Establishing specificity in psychotherapy: a meta-analysis of structural equivalence of placebo controls. $J$. Consult. Clin. Psychol. 71, 973-979.
Bishop, S. R., Lau, M., Shapiro, S., Carlson, L., Anderson, N. D., Carmody, J., Segal, Z. V., Abbey, S., Speca, M., Velting, D., and Devins, G. (2004). Mindfulness: a proposed operational definition. Clin. Psychol. Sci. Pract. 11, 230-241.

Boroojerdi, B., Bushara, K. O., Corwell, B., Immisch, I., Battaglia, F., Muellbacher, W., and Cohen, L. G. (2000). Enhanced excitability of the human visual cortex induced by short-term light deprivation. Cereb. Cortex 10, 529-524.

Boyke, J., Driemeyer, J., Gaser, C., Buechel, C., and May, A. (2008). Traininginduced brain structure changes in the elderly. J. Neurosci. 28, 7031-7035.

Brefczynski-Lewis, J. A., Lutz, A., Schaefer, H. S., Levinson, D. B., and Davidson, R. J. (2007). Neural correlates of attentional expertise in long-term meditation practition11483-11488. ers. Proc. Natl. Acad. Sci. U.S.A. 104,

learning and transfer of learning, and many of them have previously been suggested to account for the more general learning observed after action video gaming, musical training, and athletic training (Green and Bavelier, 2008; Hillman et al., 2008). Like meditation, these more "natural" training regimens are exceedingly complex, tap many systems in parallel, and include variability in task and input (Green and Bavelier, 2008). Challenging and variable training thus appears important for inducing flexible learning that more easily generalizes to novel stimuli and task contexts. A key difference between meditation and these natural training regimens is that many meditation practices are explicitly designed to target specific, well-defined core cognitive processes. This too may contribute to the ability of mental training as cultivated by meditation to induce process-specific learning.

Future research should examine which specific aspects of a particular style of meditation actually produce changes in brain and cognitive function. As mentioned above, both FA and OM meditation tap several cognitive processes in parallel. It remains to be determined which mental training-related changes in cognitive processing actually produce, e.g., the observed differences in attention and brain function reported by some of the studies reviewed above. Future research should also be directed toward investigating the unique challenges that the studies on meditation practices present in designing appropriate controls. In addition, more research should be done on the "dose response" of meditation practices to determine what may be effective study durations and to help standardize training interventions.

While the neuroscientific study of meditation is still in its infancy, mainstream psychology and cognitive neuroscience will arguably be well served by engaging in a more open, but nonetheless critical and rigorous, examination of the findings from meditation studies. Such findings may help to determine the extent to which the adult brain is plastic or subject to change, identify the basic mechanisms that underlie process-specific learning, and could lead to further exploration of cognitive-neural systems that are resilient to damage, amenable to reorganization, and capable of improving efficiency of processing through training or pharmacological treatment.

Buonomano, D. V., and Merzenich, M. M. (1998). Cortical plasticity: from synapses to maps. Annu. Rev. Neurosci. 21, 149-186.

Cahn, B. R., and Polich, J. (2006). Meditation states and traits: EEG, ERP, and neuroimaging studies. Psychol. Bull. 132, 180-211.

Carter, O. L., Presti, D. E., Callistemon, C., Ungerer, Y., Liu, G. B., and Pettigrew, J. D. (2005). Meditation alters perceptual rivalry in Tibetan Buddhist monks. Curr. Biol. 15, R412-R413.

Chambers, R., Gullone, E., and Allen, N. B. (2009). Mindful emotion regulation: an integrative review. Clin. Psychol. Rev. 29, 560-572.

Cohen, J. D., Braver, T. S., and O’Reilly, R. C. (1996). A computational approach to prefrontal cortex, cognitive control and schizophrenia: recent developments and current challenges. Philos.
Trans. R. Soc. Lond. B. Biol. Sci. 351, 1515-1527.

Colzato, L. S., Spape, M., Pannebakker, M. M., and Hommel, B. (2007). Working memory and the attentional blink: blink size is predicted by individual differences in operation span. Psychon. Bull. 14, 1051-1057.

Dahlin, E., Neely, A. S., Larsson, A., Backman, L., and Nyberg, L. (2008). Transfer of learning after updating training mediated by the striatum. Science 320, 1510-1512.

Decety, J. (1996). Neural representations for action. Rev. Neurosci. 7, 285-297.

Decety, J., Jeannerod, M., and Prablanc, C. (1989). The timing of mentally represented actions. Behav. Brain Res. 34, 35-42.

Delmonte, M. (1988). Personality correlates of meditation practice and frequency in an outpatient population. J. Behav. Med. 11, 593-597. 
Delmonte, M., and Kenny, V. (1985). Models of meditation. Br. J. Psychother. 1, 197-214.

Donohue, S. E., Woldorff, M. G., and Mitroff, S. R. (2010). Video game players show more precise multisensory temporal processing abilities. Atten. Percept. Psychophys. 72, 1120-1129.

Draganski, B., Gaser, C., Busch, V., Schuierer, G., Bogdahn, U., and May, A. (2004). Neuroplasticity: changes in grey matter induced by training newly honed juggling skills show up as a transient feature on a brain-imaging scan. Nature 427, 311-312.

Driskell, J. E., Copper, C., and Moran, A. (1994). Does mental practice enhance performance? J. Appl. Psychol. 79, 481-492.

Duncan, J., and Owen, A. M. (2000). Common regions of the human frontal lobe recruited by diverse cognitive demands. Trends Neurosci. 23, 475-483.

Ericsson, K. A. (1996). The role of deliberate practice in the acquisition and maintenance of expert performance. Int. J. Psychol. 31, 4661.

Ericsson, K. A., and Lehmann, A. C. (1996). Expert and exceptional performance: evidence of maximal adaptation to task constraints. Annu. Rev. Psychol. 47, 273-305.

Farb, N. A., Segal, Z. V., Mayberg, H., Bean, J., McKeon, D., Fatima, Z., and Anderson,A.K. (2007).Attending to the present: mindfulness meditation reveals distinct neural modes of self-reference. Soc. Cogn. Affect. Neurosci. 2, 313-322.

Formby, C., Sherlock, L. P., and Gold, S. L. (2003). Adaptive plasticity of loudness induced by chronic attenuation and enhancement of the acoustic background. J. Acoust. Soc. Am. 114, 55-58.

Frankenhaeuser, M., and Gardell, B. (1976). Underload and overload in working life - outline of a multidisciplinary approach. J. Human Stress 2, 35-46.

Fuster, J. M. (1997). The Prefrontal CortexAnatomy Physiology, Neuropsychology of the Frontal Lobe, 3rd Edn. Philadelphia: Lippincott-Raven.

Grant, J. A., Courtemanche, J., Duerden, E. G., Duncan, G. H., and Rainville, P. (2010). Cortical thickness and pain sensitivity in zen meditators. Emotion 10, 43-53

Green, C. S., and Bavelier, D. (2003).Action video game modifies visual selective attention. Nature 423, 534-537.

Green,C.S., and Bavelier, D. (2006). Effect of action video games on the spatial distribution of visuospatial attention. J. Exp. Psychol. Hum. Percept. Perform. 32, 1465-1478.

Green, C. S., and Bavelier, D. (2008). Exercising your brain: a review of human brain plasticity and traininginduced learning. Psychol. Aging 23, 692-701.

Grush, R. (2004). The emulation theory of representation: motor control, imagery, and perception. Behav. Brain Sci. 27, 377-396.

Guillot, A., and Collet, C. (2005). Contribution from neurophysiological and psychological methods to the study of motor imagery. Brain Res. Rev. 50, 387-397.

Gunaratana, H. (2007). Mindfulness in Plain English. Somerville: Wisdom Publications.

Gyatso, T., and Jinpa, T. (1995). The World of Tibetan Buddhism: an Overview of its Philosophy and Practice. Somerville: Wisdom Publications.

Hillman, C. H., Erickson, K. I., and Kramer,A.F. (2008). Be smart, exercise your heart: exercise effects on brain and cognition. Nat. Rev. Neurosci. 9, 58-65.

Hölzel, B. K., Ott, U., Gard, T., Hempel, H., Weygandt, M., Morgen, K., and Vaitl, D. (2008). Investigation of mindfulness meditation practitioners with voxel-based morphometry. Soc. Cogn. Affect. Neurosci. 3, 55-61.

Jaeggi, S. M., Buschkuehl, M., Jonides, J., and Perrig, W. J. (2008). Improving fluid intelligence with training on working memory. Proc. Natl. Acad. Sci. U.S.A. 105, 6829-6833.

Jeannerod, M. (1994). The representing brain - neural correlates of motor intention and imagery. Behav. Brain Sci. 17, 187-202.

Jeannerod, M. (1995). Mental imagery in the motor context. Neuropsychologia 33, 1419-1432.

Jeannerod, M. (1996). Motor representations: one or many? Behav. Brain Sci. 19, 763-765.

Jeffries, L. N., Smilek, D., Eich, E., and Enns, J. T. (2008). Emotional valence and arousal interact in attentional control. Psychol. Sci. 19, 290-295.

Jha, A. P., Krompinger, J., and Baime, M. J. (2007). Mindfulness training modifies subsystems of attention. Cogn. Affect. Behav. Neurosci. 7, 109-119.

Kabat-Zinn, J., Lipworth, L., and Burney, R. (2000). The clinical use of mindfulness meditation for the self-regulation of chronic pain. J. Behav. Med. 8, 163-190.

Kosslyn, S. M., Ganis, G., and Thompson, W. L. (2007). "Mental imagery and the human brain," in Progress in Psychological Science Around the World, Vol. 1, Neural, Cognitive and Developmental Issues, eds. Q. Jing, M. R. Rosenzweig, G. d'Ydewalle, H. Zhang, H.-C. Chen, and K. Zhang (New York: NY Psychology Press), 195-209.

Kramer, R. F., and Willis, S. L. (2003). "Cognitive plasticity and aging," in
Psychology of Learning and Motivation: Advances in Research and Theory, Vol. 43, ed. B. H. Ross (Amsterdam: Academic Press), 267-302.

Lacourse, M. G., Orr, E. L. R., Cramer, S. C., and Cohen, M. J. (2005). Brain activation during execution and motor imagery of novel and skilled sequential hand movements. Neuroimage 27, 505-519.

Lazar, S. W., Kerr, C. E., Wasserman, R. H., Gray, J. R., Greve, D. N., Treadway, M. T., McGarvey, M., Quinn, B. T., Dusek, J. A., Benson, H., Rauch, S. L., Moore, C. I., and Fischl, B. (2005). Meditation experience is associated with increased cortical thickness. Neuroreport 16, 1893-1897.

Leung, Y, and Singhal,A. (2004).An examination of the relationship between qigong meditation and personality. Soc. Behav. Pers. 32, 313-320.

Lotze, M., Montoya, P., Erb, M., Hülsmann, E., Flor, H., Klose, U., Birbaumer, N., and Grodd, W. (1999). Activation of cortical and cerebellar motor areas during executed and imagined hand movements: an fMRI study. J. Cogn. Neurosci. 11, 491-501.

Lutz, A., Dunne, J. D., and Davidson, R. J. (2007). "Meditation and the neuroscience of consciousness: an introduction," in Cambridge Handbook of Consciousness, eds P. Zelazo, M. Moscovitch, and E. Thompson (New York: Cambridge University Press), 81-109.

Lutz, A., Slagter, H. A., Rawlings, N. B., Francis, A. D., Greischar, L. L., and Davidson, R. J. (2009). Mental training enhances attentional stability: neural and behavioral evidence. J. Neurosci. 29, 13418-13427.

Lutz, A., Greischar, L. L., Rawlings, N. B., Ricard, M., and Davidson, R. J. (2004). Long-term meditators selfinduce high-amplitude gamma synchrony during mental practice. Proc. Natl. Acad. Sci. U.S.A. 101 16369-16373.

Lutz, A., Slagter, H. A., Dunne, J. D., and Davidson, R. J. (2008). Attention regulation and monitoring in meditation. Trends Cogn. Sci. 12, 163-169.

MacCoon, D. G., Sullivan, J. C., Davidson, R. J., Stoney, C. M., Johnson, L. L., Christmas, P. D., and Lutz, A. (2011). Health-Enhancement Program (HEP) Guidelines. Available at: http://digital. library.wisc.edu/1793/28198.

MacLean, K. A., Ferrer, E., Aichele, S. R. Bridwell, D. A., Zanesco, A. P., Jacobs, T. L., King, B. G., Rosenberg, E. L., Sahdra, B. K., Shaver, P. R., Wallace, B. A., Mangun, G. R., and Saron, C. D. (2010). Intensive meditation training improves perceptual discrimination and sustained attention. Psychol. Sci. 21, 829-839.
Maguire, E. A., Gadian, D. G., Johnsrude, I. S., Good, C. D., Ashburner, J., Frackowiak, R. S., and Frith, C. D. (2000). Navigation-related structural change in the hippocampi of taxi drivers. Proc. Natl. Acad. Sci. U.S.A. 97, 4398-4403.

Maguire, E. A., Woollett, K., and Spiers, H. J. (2006). London taxi drivers and bus drivers: a structural MRI and neuropsychological analysis. Hippocampus 16, 1091-1101.

Mahon, B. Z., and Caramazza, A. (2008). A critical look at the embodied cognition hypothesis and a new proposal for grounding conceptual content. J. Physiol. 102, 59-70.

Minas, S. C. (1978). Mental practice of a complex perceptual motor skill. J. Hum. Mov. Stud. 4, 102-109.

Miyake, A., Friedman, N. P., Emerson, M. J., Witzki, A. H., Howerter, A., and Wager, T. D. (2000). The unity and diversity of executive functions and their contributions to complex "Frontal Lobe" tasks: a latent variable analysis. Cogn. Psychol. 41, 49-100.

Munro, K. J., and Blount, J. (2009). Adaptive plasticity in brainstem of adult listeners following earpluginduced deprivation. J. Acoust. Soc. Am. 126, 568-571.

Munzert, J., Lorey, B., and Zentgraf, K. (2009). Cognitive motor processes: the role of motor imagery in the study of motor representations. Brain Res. Rev. 60, 306-326.

Nyberg, L., Eriksson, J., Larsson, A., and Marklund,P.(2006).Learning by doing versus learning by thinking: an fMRI study of motor, and mental training. Neuropsychologia 44, 711-717.

Olesen, P.J., Westerberg, H., and Klingberg, T. (2004). Increased prefrontal and parietal activity after training of working memory. Nat. Neurosci. 7, 75-79.

Olivers, C. N., and Nieuwenhuis, S. (2006). The beneficial effects of additional task load positive affect and instruction on the attentional blink. J. Exp. Psychol. Hum. Percept. Perform. 32, 364-379.

Olsson, C. J., Jonsson, B., and Nyberg, L. (2008). Learning by doing and learning by thinking: an fMRI study of combining motor and mental training. Front. Hum. Neurosci. 2:5. doi: 10.3389/neuro.09.005.2008

Pascual-Leone, A., Amedi, A., Fregni, F., and Merabet, L. B. (2005). The plastic human brain cortex. Annu. Rev. Neurosci. 28, 377-401.

Pascual-Leone, A., Nguyet, D., Cohen, L. G., Brasil-Neto, J. P., Cammarota, A., and Hallett, M. (1995). Modulation of muscle responses evoked by transcranial magnetic stimulation during the acquisition of new fine motor skills. J. Neurophysiol. 74, 1037-1045. 
Persson, J., and Reuter-Lorenz, P. A. (2008). Gaining control training executive function and far transfer of the ability to resolve interference. Psychol. Sci. 19, 881-888.

Pfurtscheller, G., and da Silva, F. H. L. (1999). Event-related EEG/MEG synchronization and desynchronization: basic principles. Clin. Neurophysiol. 110, 1842-1857.

Pitskel, N. B., Merabet, L. B., RamosEstabanez, C., Kauffman, T., and Pascual-Leone, A. (2007). Timedependent changes in cortical excitability after prolonged visual deprivation. Neuroreport 18, 1703-1707.

Posner, M. I., and Petersen, S. E. (1990). The attention system of the human brain. Annu. Rev. Neurosci. 13, 25-42.

Ranganathan, V. K., Siemionow, V., Liu, J. Z., Sahgal, V., and Yue, G. H. (2004). From mental power to muscle powergaining strength by using the mind. Neuropsychologia 42, 944-956.

Roelfsema, P. R., van Ooyen, A., and Watanabe, T. (2010). Perceptual learning rules based on reinforcers and attention. Trends Cogn. Sci. 14, 64-71.

Romero, S. G., McFarland, D. J., Faust, R., Farrell, L., and Cacace, A. T. (2008). Electrophysiological markers of skillrelated neuroplasticity. Biol. Psychol. 78, 221-230.

Sahdra, B. K., MacLean, K. A., Ferrer, E., Shaver, P. R., Rosenberg, E. L., Jacobs,
T. L., Zanesco, A. P., Aichele, S. R., King, B. G., Bridwell, D. A., Lavy, S., Mangun, G. R., Wallace, A. B., and Saron, C. D. (in press). Enhanced response inhibition during intensive meditation training predicts improvements in self-reported adaptive socioemotional functioning. Emotion.

Salomon, G., and Perkins, D. N. (1989). Rocky roads to transfer - rethinking mechanisms of a neglected phenomenon. Educ. Psychol. 24, 113-142.

Schmidt, R. A., and Bjork, R. A. (1992). New conceptualizations of practice common principles in 3 paradigms suggest new concepts for training. Psychol. Sci. 3, 207-217.

Seidler, R. D. (2004). Multiple motor learning experiences enhance motor adaptability. J. Cogn. Neurosci. 16, 65-73.

Slagter, H. A., Lutz, A., Greischar, L. L. Francis, A. D., Nieuwenhuis, S., Davis, J. M., and Davidson, R. J. (2007). Mental training affects distribution of limited brain resources. PLoS Biol. 5, 1228-1235. doi: 10.1371/journal. pbio. 0050138

Slagter, H. A., Lutz, A., Greischar, L. L., Nieuwenhuis, S., and Davidson, R. J. (2009). Theta phase synchrony and conscious target perception: impact of intensive mental training. J. Cogn. Neurosci. 21, 1536-1549.

Smith, E. E., and Jonides, J. (1999). Storage and executive processes in the frontal lobes. Science 283, 1657-1661.
Suinn, R. M. (1984). "Imagery and sports," in Cognitive Sport Psychology, eds W. Straub and J. Williams (Lansing, NY: Sport Science Associates).

Takahashi, T., Murata, T., Hamada, T. Omori, M., Kosaka, H., Kikuchi, M. Yoshida, H., and Wada, Y. (2005). Changes in EEG and autonomic nervous activity during meditation and their association with personality traits. Int. J. Psychophysiol. 55, 199-207.

Tang, Y. Y., Lu, Q., Geng, X., Stein, E. A., Yang, Y., and Posner, M. I. (2010). Short-term meditation induces white matter changes in the anterior cingulate. Proc. Natl. Acad. Sci. U.S.A. 107, 15649-15652.

Tang, Y. Y., Ma, Y., Wang, J., Fan, Y., Feng, S., Lu, Q., Yu, Q., Sui, D., Rothbart, M. K., Fan, M., and Posner, M. I. (2007). Short-term meditation training improves attention and self-regulation. Proc. Natl. Acad. Sci. U.S.A. 104, 17152-17156.

Tang, Y. Y., and Posner, M. I. (2009). Attention training and attention state training. Trends Cogn. Sci. 13, 222-227.

Teasdale, J. D., Segal, Z. V., Williams, J. M., Ridgeway, V. A., Soulsby, J. M., and Lau, M. A. (2000). Prevention of relapse/recurrence in major depression by mindfulness-based cognitive therapy. J. Consult. Clin. Psychol. 68, 615-623.
Wohldmann, E. L., Healy, A. F., and Bourne, L.E. (2008). A mental practice superiority effect: less retroactive interference and more transfer than physical practice. J. Exp. Psychol. Learn. Mem. Cogn. 34, 823-833.

Woollett, K., and Maguire, E. A. (2009). Navigational expertise may compromise anterograde associative memory. Neuropsychologia 47, 1088-1095.

Xiong, G. L., and Doraiswamy, P. M. (2009). Does meditation enhance cognition and brain plasticity? Ann. N. Y. Acad. Sci. 1172, 63-69.

Conflict of Interest Statement: The authors declare that the research was conducted in the absence of any commercial or financial relationships that could be construed as a potential conflict of interest.

Received:07 July 2010; accepted: 26 January 2011; published online: 10 February 2011. Citation: Slagter HA, Davidson RJ and Lutz A (2011) Mental training as a tool in the neuroscientific study of brain and cognitive plasticity. Front. Hum. Neurosci. 5:17. doi:10.3389/fnhum.2011.00017

Copyright (c) 2011 Slagter, Davidson and Lutz. This is an open-access article subject to an exclusive license agreement between the authors and Frontiers Media SA, which permits unrestricted use, distribution, and reproduction in any medium, provided the original authors and source are credited. 